\title{
Equilibrium Existence in Global Games With General Payoff Structures
}

\author{
By
}

\author{
Eric J. Hoffmann* and Tarun Sabarwal ${ }^{\dagger}$
}

\begin{abstract}
We consider global games with general payoff structures and prove existence of equilibrium. This shows that the global games method is well-defined with arbitrary strategic interaction among players, thus providing a foundation for the study of more general equilibrium behavior, especially as research in global games moves beyond the case of strategic complements. We also show that in every global game, even though the information of each player is correlated, the joint information measure is absolutely continuous with respect to the product of its marginals. As one application, the result here can be used to show existence of equilibrium in global games with both complementarity and congestion. This proves existence of equilibrium in a finiteplayer version of the model in Karp, Lee, and Mason (2007), thus addressing a gap in the proof of equilibrium existence documented in Hoffmann and Sabarwal (2015).
\end{abstract}

JEL Numbers: C70, C72, C73

Keywords: Global games, strategic complements, strategic substitutes, strategic heterogeneity, monotone games, equilibrium selection

First Draft: April 2014

This Version: November 13, 2017

\footnotetext{
*Department of Economics, West Texas A \& M University. Email: ehoffmann@wtamu.edu.
}

${ }^{\dagger}$ Department of Economics, University of Kansas. Email: sabarwal@ku.edu. 


\section{Introduction}

Global games (Carlsson and Van Damme (1993)) provide a way to think about equilibrium selection and robustness of an equilibrium in a complete information game when we add a small amount of uncertainty. Intuitively, in the presence of uncertainty, equilibrium actions in some environments can affect equilibrium actions in other relatively remote environments through a contagion argument. This approach has been used successfully to understand several important economic phenomenon, including speculative attacks in currency markets, investment decisions, and macroeconomic coordination failures. There is a large literature in this area. For a survey of theory and applications, confer Morris and Shin (2003). ${ }^{1}$

The global games approach has been extended in many directions, including finitely many players (as one example, confer Frankel, Morris, and Pauzner (2003)), a continuum of symmetric players, as in Morris and Shin (2003), finitely many actions, as in Oury (2013), actions in [0,1], as in Frankel, Morris, and Pauzner (2003), and to multidimensional uncertainty, as in Oury (2013).

All these approaches make assumptions on payoff structures that yield strategic complements in actions across players. These assumptions typically include some form of supermodularity, increasing differences, and state monotonicity. Once we allow more gen-

\footnotetext{
${ }^{1}$ For a small sampling of the literature, also confer Frankel, Morris, and Pauzner (2003), Heinemann, Nagel, and Ockenfels (2004), Angeletos, Hellwig, and Pavan (2006), Steiner and Stewart (2008), Basteck, Daniëls, and Heinemann (2013), and Oury (2013), among others.
} 
eral payoff structures, there do not appear to be general results that show existence of an equilibrium in global games. This is due to several reasons.

First, in general, once strategic substitutes are introduced, a complete information game may not necessarily have a pure-strategy Nash equilibrium (confer Roy and Sabarwal (2012)) and similarly, a game with both strategic complements and strategic substitutes may not necessarily have a pure-strategy Nash equilibrium (confer Monaco and Sabarwal $(2016))$.

Second, models using an improper prior in global games (as in Morris and Shin (2003)) do not have a prior distribution of uncertainty in the normal sense of a probability, and in these cases, it is hard to connect a global game with the standard model of an incomplete information game. This makes it harder to invoke standard results on existence of equilibrium in an incomplete information game.

Third, the information structure assumed in a global game makes it hard to apply a standard proof of existence of equilibrium, because the observation of each player is correlated with that of every other player (they all depend on the same underlying state of the world). This correlation can potentially jeopardize an important assumption (requiring absolute continuity of the joint information measure with respect to the product of its marginals) in the general proofs of existence of an equilibrium in games of incomplete information (such as in Balder (1988) and Milgrom and Weber (1985)).

A main contribution of the work here is to formulate a model of a global game that 
allows for arbitrary payoff structures (with only mild assumptions related to continuity and integrability) and to show existence of equilibrium in this general model. This generalizes existing models of global games by allowing for arbitrary strategic heterogeneity across players. In the process, we also show that in every global game, even though the information of each player is correlated with that of other players, the joint information measure is absolutely continuous with respect to the product of its marginals.

As interest in global games moves beyond cases with strategic complements, our result provides a general framework in which the global games method is well-defined. This provides a foundation for the study of equilibrium behavior in global games with strategic heterogeneity. As one application, the results here can be used to show that there is a global game equilibrium in the finite-player version of the model in Karp, Lee, and Mason (2007).

This paper focuses on existence of equilibrium in a global game. The important problems of uniqueness of the global games equilibrium selection (sometimes termed limit uniqueness) in terms of dominance solvability, and its uniqueness as noise goes to zero (sometimes termed noise independence) remain open in the general case. Showing that an equilibrium exists in the more general case provides a first step to other researchers in this regard.

The next section formalizes a model of a global game and defines a global game equilibrium. The section after that proves existence of equilibrium in every global game represented by this model. It also includes some extensions and discussion. The last 
section concludes.

\section{Global Game and Equilibrium}

Consider a finite collection of players, indexed $i=1, \ldots, N$. The action space for player $i$ is denoted $A_{i}$, where $A_{i}$ is assumed to be a compact metric space, ${ }^{2}$ and its Borel sigma-algebra is denoted $\mathcal{A}_{i}$. The product spaces are denoted $A=\times_{i=1}^{N} A_{i}$, and $\mathcal{A}=\times_{i=1}^{N} \mathcal{A}_{i}$

Following the standard motivation for a global game, suppose there is a payoff relevant state of the world and each player observes this state with some uncertainty. The structure of uncertainty in a global game is formalized as follows. Let $\varepsilon_{0}$ be a random variable governing the behavior of the underlying state of the world. It is distributed on an open set $X_{0} \subset \mathbb{R}$ with density $\phi_{0}$ having full support. ${ }^{3}$ Given an underlying state of the world $x_{0} \in X_{0}$, each player $i \in\{1, \ldots, N\}$ observes $x_{i}=x_{0}+\varepsilon_{i}$, where $\varepsilon_{i}$ are distributed on $[-\epsilon, \epsilon]$ (for some small $\epsilon>0$ ) with density $\phi_{i}$ having full support, ${ }^{4}$ and $\left(\varepsilon_{i}\right)_{i=0}^{N}$ are jointly independent. Let $T_{0}=(\underline{x}-\epsilon, \bar{x}+\epsilon)$, where $\underline{x}, \bar{x}$ determine dominance

\footnotetext{
${ }^{2}$ This is more general than the standard assumption in global games, which typically assume finitely many or a continuum of actions.

${ }^{3}$ As usual, we take the Borel sigma-algebra and Lebesgue measure on $\mathbb{R}$. Moreover, modifying $\phi_{0}$ on a set of Lebesgue measure zero if necessary, suppose that for every $x_{0} \in X_{0}, \phi_{0}\left(x_{0}\right)>0$.

${ }^{4}$ Again, as usual, we take the Borel sigma-algebra and Lebesgue measure on $\mathbb{R}$ and restrict it to $[-\epsilon, \epsilon]$. Moreover, modifying $\phi_{i}$ on a set of Lebesgue measure zero if necessary, suppose that for every $\xi \in[-\epsilon, \epsilon]$, $\phi_{i}(\xi)>0$.
} 
regions, as defined below, and assume that $T_{0} \subset \operatorname{support}\left(\phi_{0}\right) .^{5}$

The payoff of each player $i=1, \ldots, N$ in a global game depends on the underlying state of the world $x_{0} \in X_{0} \subset \mathbb{R}$ and on the actions of players $1, \ldots, N$, and is given by a function $U_{i}: X_{0} \times A \rightarrow \mathbb{R}$, denoted $U_{i}\left(x_{0}, a_{1}, \ldots, a_{N}\right)$. We assume that for every player $i \in\{1, \ldots, N\}, U_{i}$ is $\mathcal{X}_{0} \times \mathcal{A}$-measurable on $X_{0} \times A$, where $\mathcal{X}_{0}$ denotes the Borel sigmaalgebra on $X_{0}$. Moreover, (for the smaller set $T_{0} \subset X_{0}$,) we assume that for every $x_{0} \in T_{0}$, $U_{i}\left(x_{0}, \cdot\right)$ is continuous on $A$, and that $\left|U_{i}\right| \leq \Phi_{i}$ on $T_{0} \times A$ for some $\Phi_{i} \in \mathcal{L}_{1}\left(T_{0}, \mathcal{T}_{0}, \tilde{\phi}_{0}\right)$, where $\mathcal{T}_{0}$ is the Borel sigma-algebra on $T_{0}, \tilde{\phi}_{0}$ is the normalized density on $T_{0}$ derived from $\phi_{0}$, as usual, by setting $\tilde{\phi}_{0}=\frac{1}{\int_{T_{0}} \phi_{0}\left(x_{0}\right) \lambda\left(d x_{0}\right)} \phi_{0}$ on $T_{0}$ and zero otherwise, and $\mathcal{L}_{1}\left(T_{0}, \mathcal{T}_{0}, \tilde{\phi}_{0}\right)$ is the corresponding space of integrable functions.

The payoffs here are very general with only mild assumptions related to continuity, measurability, and integrability. In particular, there are no requirements related to supermodularity, increasing differences, or state monotonicty. This allows for arbitrary strategic heterogeneity across players.

Moreover, the continuity and integrability conditions are only required on $T_{0} \times A$ and not the potentially larger $X_{0} \times A$. This allows for cases where payoffs are integrable when realizations of state of the world are restricted to the smaller set $T_{0}$ but not necessarily on the possibly unbounded larger set $X_{0}$.

Dominance regions in a global game are assumed as follows. There are real numbers $\underline{x}<\bar{x}$ such that for $x_{0}<\underline{x}$ the strictly dominant action for each player $i \in\{1, \ldots, N\}$ is

\footnotetext{
${ }^{5}$ In particular, this implies that $\int_{T_{0}} \phi_{0}\left(x_{0}\right) \lambda\left(d x_{0}\right)>0$, where $\lambda$ is Lebesgue measure on $\mathbb{R}$.
} 
to play $\underline{a}_{i} \in A_{i}$ and for $x_{0}>\bar{x}$ the strictly dominant action for each player $i$ is to play $\bar{a}_{i} \in A_{i}$. Here, $\underline{a}_{i}$ and $\bar{a}_{i}$ are arbitrarily fixed actions in $A_{i}$. The dominance regions are denoted $D_{1}=(-\infty, \underline{x}]$ and $D_{2}=[\bar{x}, \infty)$.

A global game is a collection $\Gamma=\left(\left(A_{i}, \mathcal{A}_{i}\right)_{i=1}^{N},\left(\varepsilon_{i}\right)_{i=0}^{N},\left(U_{i}\right)_{i=1}^{N},\left(D_{1}, D_{2}\right)\right)$, with finitely many players, indexed $i=1, \ldots, N$, where $\left(A_{i}, \mathcal{A}_{i}\right)_{i=1}^{N}$ are action spaces for the players, $\left(\varepsilon_{i}\right)_{i=0}^{N}$ formalizes the structure of uncertainty, $\left(U_{i}\right)_{i=1}^{N}$ are payoffs, and $\left(D_{1}, D_{2}\right)$ are dominance regions.

In a global game, the joint information structure is defined as follows. For ease of notation, we introduce player 0 ("chance" or "nature") with type space $X_{0}$ as above, endowed with the Borel sigma-algebra $\mathcal{X}_{0}$, and density $\phi_{0}$. The type space of players $i=$ $1, \ldots, N$ is $X_{i}=\left\{x_{0}+\xi \in \mathbb{R}: x_{0} \in X_{0}\right.$ and $\left.\xi \in[-\epsilon, \epsilon]\right\}$ with the Borel sigma-algebra $\mathcal{X}_{i}$. The joint type space is given by $X=\times_{i=0}^{N} X_{i}$ with the product sigma-algebra $\mathcal{X}=\times_{i=0}^{N} \mathcal{X}_{i}$. The joint density on $X$ is given by $\phi\left(x_{0}, x_{1}, \ldots, x_{N}\right)=\phi_{0}\left(x_{0}\right) \phi_{1}\left(x_{1}-x_{0}\right) \cdots \phi_{N}\left(x_{N}-x_{0}\right)$, so that the measure governing the random behavior of joint information on $\mathcal{X}$, denoted $\eta$, is given by $\eta(B)=\int_{B} \phi(x) \lambda^{N+1}(d x)$, where $\lambda^{N+1}$ is Lebesgue measure on $\mathbb{R}^{N+1}$ and $B \in \mathcal{X}$. The joint information structure is $\left(\left(X_{i}, \mathcal{X}_{i}\right)_{i=0}^{N}, \eta\right)$.

A behavioral strategy for player $i=1, \ldots, N$ is a function $s_{i}: X_{i} \times \mathcal{A}_{i} \rightarrow[0,1]$ such that (1) for every $x_{i} \in X_{i}, s_{i}\left(x_{i}, \cdot\right)$ is a probability measure on $\mathcal{A}_{i}$, and (2) for every Borel set $B \in \mathcal{A}_{i}, s_{i}(\cdot, B)$ is $\mathcal{X}_{i}$-measurable on $X_{i}$. The set of such strategies is denoted 
$\mathcal{S}_{i}$. Given a profile of strategies $\left(s_{1}, \ldots, s_{N}\right)$, the expected payoff to player $i=1, \ldots, N$ is

$$
\mathcal{U}_{i}\left(s_{1}, \ldots, s_{N}\right)=\int_{X}\left[\int_{A_{1}} \ldots \int_{A_{N}} U_{i}\left(x_{0}, a\right) s_{1}\left(x_{1} ; d a_{1}\right) \ldots s_{N}\left(x_{N} ; d a_{N}\right)\right] \eta(d x) .
$$

An equilibrium in a global game $\Gamma$ is a profile of behavioral strategies $\left(s_{1}^{*}, \ldots, s_{N}^{*}\right)$ such that for every player $i=1, \ldots, N, s_{i}^{*}$ is a best response of player $i$ to the strategies of the other players $s_{-i}^{*}$.

\section{Existence of Equilibrium}

Notice that an equilibrium in a global game is a Bayesian-Nash equilibrium in the related incomplete information game. Using dominance regions, we may further reduce the search for a global game equilibrium to a search for a Bayesian-Nash equilibrium with restricted type spaces, as follows.

Consider $T_{0}=(\underline{x}-\epsilon, \bar{x}+\epsilon)$ along with its Borel sigma-algebra $\mathcal{T}_{0}$, as above, and consider the normalized density $\tilde{\phi}_{0}$ on $T_{0}$ derived from $\phi_{0}$, as usual, by setting $\tilde{\phi}_{0}=$ $\frac{1}{\int_{T_{0}} \phi_{0}\left(x_{0}\right) \lambda\left(d x_{0}\right)} \phi_{0}$ on $T_{0}$ and zero otherwise. As $T_{0} \subset \operatorname{support}\left(\phi_{0}\right)$, we may suppose that for $x_{0} \in T_{0}, \phi_{0}\left(x_{0}\right)>0$, and therefore, $\int_{T_{0}} \phi_{0}\left(x_{0}\right) \lambda\left(d x_{0}\right)>0$. It follows that for $x_{0} \in T_{0}, \tilde{\phi}_{0}\left(x_{0}\right)>0$. Define the restricted type space of player $i=1, \ldots, N$ by $T_{i}=$ $\left\{x_{0}+\xi \in \mathbb{R}: x_{0} \in T_{0}\right.$ and $\left.\xi \in[-\epsilon, \epsilon]\right\}=(\underline{x}-2 \epsilon, \bar{x}+2 \epsilon)$ with the Borel sigma-algebra $\mathcal{T}_{i}$. The restricted joint type space is given by $T=\times_{i=0}^{N} T_{i}$ with the product sigmaalgebra $\mathcal{T}=\times_{i=0}^{N} \mathcal{T}_{i}$. The restricted joint density on $T$ is given by $\tilde{\phi}\left(x_{0}, x_{1}, \ldots, x_{N}\right)=$ $\tilde{\phi}_{0}\left(x_{0}\right) \phi_{1}\left(x_{1}-x_{0}\right) \cdots \phi_{N}\left(x_{N}-x_{0}\right)$, so that the measure governing the random behavior of 
joint information on $\mathcal{T}$, denoted $\tilde{\eta}$, is given by $\tilde{\eta}(B)=\int_{B} \tilde{\phi}(x) \lambda^{N+1}(d x)$, where $\lambda^{N+1}$ is Lebesgue measure on $\mathbb{R}^{N+1}$ and $B \in \mathcal{T}$. The restricted joint information structure is $\left(\left(T_{i}, \mathcal{T}_{i}\right)_{i=0}^{N}, \tilde{\eta}\right)$.

In a global game $\Gamma$, a restricted behavioral strategy for player $i=1, \ldots, N$ is a function $\tilde{s}_{i}: T_{i} \times \mathcal{A}_{i} \rightarrow[0,1]$ such that $(1)$ for every $x_{i} \in T_{i}, \tilde{s}_{i}\left(x_{i}, \cdot\right)$ is a probability measure on $\mathcal{A}_{i}$, and (2) for every Borel set $B \in \mathcal{A}_{i}, \tilde{s}_{i}(\cdot, B)$ is $\mathcal{T}_{i}$-measurable on $T_{i}$. The set of such strategies is denoted $\tilde{\mathcal{S}}_{i}$. Given a profile of restricted strategies $\left(\tilde{s}_{1}, \ldots, \tilde{s}_{N}\right)$, the expected payoff to player $i=1, \ldots, N$ is

$$
\tilde{\mathcal{U}}_{i}\left(\tilde{s}_{1}, \ldots, \tilde{s}_{N}\right)=\int_{T}\left[\int_{A_{1}} \ldots \int_{A_{N}} U_{i}\left(x_{0}, a\right) s_{1}\left(x_{1} ; d a_{1}\right) \ldots s_{N}\left(x_{N} ; d a_{N}\right)\right] \tilde{\eta}(d x)
$$

The restricted incomplete information game is given by $\left(\left(\tilde{\mathcal{U}}_{i}, \tilde{\mathcal{S}}_{i}, T_{i}, \mathcal{T}_{i}\right)_{i=0}^{N}, \tilde{\eta}\right)$, with $N+1$ players, where as usual, we may add player 0 as "chance" with type space given by $\left(T_{0}, \mathcal{T}_{0}\right)$, a singleton strategy space, and payoffs set identically to zero (therefore, no strategic motive). A Bayesian-Nash equilibrium in the restricted incomplete information game can be extended to a global game equilibrium, as follows.

Theorem 1. Let $\Gamma$ be a global game and $\left(\left(\tilde{\mathcal{U}}_{i}, \tilde{\mathcal{S}}_{i}, T_{i}, \mathcal{T}_{i}\right)_{i=0}^{N}, \tilde{\eta}\right)$ be the corresponding restricted incomplete information game.

Every Bayesian-Nash equilibrium in the restricted incomplete information game can be extended to a global game equilibrium in $\Gamma$.

Proof. Suppose $\left(\tilde{s}_{0}^{*}, \tilde{s}_{1}^{*}, \ldots, \tilde{s}_{N}^{*}\right)$ is a Bayesian-Nash equilibrium in the restricted incomplete information game. For each player $i=1, \ldots, N$, extend $\tilde{s}_{i}^{*}$ to $s_{i}^{*}$ on $X_{i} \times \mathcal{A}_{i}$, as 
follows. For $x_{i} \in T_{i}$, let $s_{i}^{*}\left(x_{i}, \cdot\right)=\tilde{s}_{i}^{*}\left(x_{i}, \cdot\right)$. Now consider $x_{i} \in X_{i} \backslash T_{i}$. As case 1 , suppose that $x_{i}$ is a lower bound for $T_{i}=(\underline{x}-2 \epsilon, \bar{x}+2 \epsilon)$. Then $x_{i}$ is in the dominance region $D_{1}$ for player $i$, and the strictly dominant action for player $i$ in this region is $\underline{a}_{i}$. Let $s_{i}^{*}\left(x_{i}, \cdot\right)$ be the degenerate probability measure on $\underline{a}_{i}$. As case 2 , suppose that $x_{i}$ is an upper bound for $T_{i}=(\underline{x}-2 \epsilon, \bar{x}+2 \epsilon)$. Then $x_{i}$ is in the dominance region $D_{2}$ for player $i$, and the strictly dominant action for player $i$ in this region is $\bar{a}_{i}$. Let $s_{i}^{*}\left(x_{i}, \cdot\right)$ be the degenerate probability measure on $\bar{a}_{i}$. Consider the strategy profile $\left(0, s_{1}^{*}, \ldots, s_{N}^{*}\right)$. It must be that for every $i=0,1, \ldots, N, s_{i}^{*}$ is a best response to $s_{-i}^{*}$. (Otherwise, it is not a best response for some $i=1, \ldots, N$. In this case, we may modify $s_{i}^{*}$ on a set of positive measure and do better against $s_{-i}^{*}$. This set of positive measure may be taken to be a subset of $T_{i}$, because on its complement, player $i$ is playing strictly dominant actions. Using the same modification for $\tilde{s}_{i}^{*}$ and the fact that $\tilde{\phi}$ is a positive scalar multiple of $\phi$ yields a violation of the fact that $\tilde{s}_{i}^{*}$ is a best response to $\tilde{s}_{-i}^{*}$.) Therefore, $\left(s_{1}^{*}, \ldots, s_{N}^{*}\right)$ is a global game equilibrium.

This result reduces the problem of finding a global game equilibrium to that of finding a Bayesian-Nash equilibrium in the corresponding restricted incomplete information game. This can be done as follows.

Notice that the information structure in a global game has the property that information of each player is correlated with that of every other player, because they all depend on the realization of the same underlying state of the world $x_{0}$. In particular, if there is a higher realization of state of the world, then each player's observation is distributed over 
a higher region as well.

This correlation can potentially violate an important assumption (requiring absolute continuity of the joint information measure with respect to the product of its marginals) required in the general proofs of existence of an equilibrium in games of incomplete information (such as in Balder (1988) and Milgrom and Weber (1985)). ${ }^{6}$ Recall that when there is correlation, a joint information measure is not necessarily absolutely continuous with respect to the product of its marginals. For example, consider the unit square $[0,1] \times[0,1]$ and the joint measure given by the uniform distribution on the diagonal. In this case, each marginal measure is given by the uniform distribution on $[0,1]$. The product of the marginals assigns measure zero to the diagonal, but the joint measure assigns measure 1 to the diagonal.

We show that the presence of correlation in the information structure of a global game does not violate this assumption, that is, the measure $\tilde{\eta}$ in the restricted joint information structure $\left(\left(T_{i}, \mathcal{T}_{i}\right)_{i=0}^{N}, \tilde{\eta}\right)$ is absolutely continuous with respect to the product of the marginals derived from $\tilde{\eta}$.

Theorem 2. Let $\Gamma$ be a global game and $\left(\left(T_{i}, \mathcal{T}_{i}\right)_{i=0}^{N}, \tilde{\eta}\right)$ be the corresponding restricted information structure. The joint measure $\tilde{\eta}$ is absolutely continous with respect to the product of its marginals.

Proof. Recall that $\tilde{\eta}(B)=\int_{B} \tilde{\phi}(x) \lambda^{N+1}(d x)$, where $\tilde{\phi}\left(x_{0}, x_{1}, \ldots, x_{N}\right)=\tilde{\phi}_{0}\left(x_{0}\right) \phi_{1}\left(x_{1}-\right.$

\footnotetext{
${ }^{6}$ Indeed, Karp, Lee, and Mason (2007) mention this violation to motivate their alternative approach (confer page 160 of their paper).
} 
$\left.x_{0}\right) \cdots \phi_{N}\left(x_{N}-x_{0}\right)$ on $T=\times_{i=0}^{N} T_{i}, \lambda^{N+1}$ is Lebesgue measure on $\mathbb{R}^{N+1}$ and $B \in \mathcal{T}$. For each $i=0,1, \ldots, N$, let the marginal density on $T_{i}$ derived from $\tilde{\phi}$ be given by $f_{i}\left(x_{i}\right)=\int \tilde{\phi}\left(x_{i}, x_{-i}\right) \lambda^{N}\left(d x_{-i}\right)$, where $\lambda^{N}$ is Lebesgue measure on $\mathbb{R}^{N}$, and the corresponding marginal measure is given by $\mu_{i}(B)=\int_{B} f_{i}\left(x_{i}\right) \lambda(d x)$, where $\lambda$ is Lebesgue measure on $\mathbb{R}$ and $B \in \mathcal{T}_{i}$. Let the product of the marginal measures be denoted $\mu=\mu_{0} \times \mu_{1} \times \cdots \times \mu_{N}$ and the product of the marginal densities be denoted $f(x)=f_{0}\left(x_{0}\right) f_{1}\left(x_{1}\right) \cdots f_{N}\left(x_{N}\right)$. Thus, $\mu(B)=\int_{B} f(x) \lambda^{N+1}(d x)$.

To see that $\tilde{\eta}$ is absolutely continuous with respect to $\mu$, we first show that $\operatorname{support}(\tilde{\phi}) \subset$ support $(f)$, as follows. It is immediate from the definition of an information structure that $\operatorname{support}(\tilde{\phi}) \subset T=\times_{i=0}^{N} T_{i}$. The inclusion $T \subset \operatorname{support}(f)$ can be seen as follows.

For $i=0$, and for every $x_{0} \in T_{0}, f_{0}\left(x_{0}\right)=\tilde{\phi}_{0}\left(x_{0}\right)=\frac{1}{\int_{T_{0}} \phi_{0}\left(x_{0}\right) \lambda\left(d x_{0}\right)} \phi_{0}\left(x_{0}\right)>0$, because $T_{0} \subset \operatorname{support}\left(\phi_{0}\right)$ and $\int_{T_{0}} \phi_{0}\left(x_{0}\right) \lambda\left(d x_{0}\right)>0$. Moreover, it can be seen that for each player $i \in\{1, \ldots, N\}$, and for every $x_{i} \in T_{i}, f_{i}\left(x_{i}\right)>0$, as follows. Fix $x_{i} \in T_{i}$ arbitrarily, and notice that Fubini's theorem implies that $f_{i}\left(x_{i}\right)=\int \phi\left(x_{i}, x_{-i}\right) \lambda^{N}\left(d x_{-i}\right)=\int_{T_{0}} \tilde{\phi}_{0}(x) \phi_{i}\left(x_{1}-\right.$ $x) \lambda(d x)$. As $x_{i} \in T_{i}$, let $x_{0} \in T_{0}$ and $\xi \in[-\epsilon, \epsilon]$ be such that $x_{i}=x_{0}+\xi$. As case 1 , suppose $\xi \in(-\epsilon, \epsilon)$. In this case, $\left|x_{i}-x_{0}\right|<\epsilon$. As $T_{0}$ is open and absolute value is a continuous function, there is $\delta>0$ such that $\left[x_{0}-\delta, x_{0}+\delta\right] \subset T_{0}$ and for every $x \in\left[x_{0}-\delta, x_{0}+\delta\right]$, $\left|x_{i}-x\right|<\epsilon$. In other words, for every $x \in\left[x_{0}-\delta, x_{0}+\delta\right] \subset T_{0}, x_{i}-x \in[-\epsilon, \epsilon]$. Using full support of $\phi_{i}$, it follows that for every $x \in\left[x_{0}-\delta, x_{0}+\delta\right] \subset T_{0}, \phi_{0}(x) \phi_{i}\left(x_{i}-x\right)>0$. Consequently, $f_{i}\left(x_{i}\right)=\int_{T_{0}} \tilde{\phi}_{0}(x) \phi_{i}\left(x_{1}-x\right) \lambda(d x) \geq \int_{\left[x_{0}-\delta, x_{0}+\delta\right]} \tilde{\phi}_{0}(x) \phi_{i}\left(x_{1}-x\right) \lambda(d x)>0$. As case 2, suppose $\xi=-\epsilon$. In this case, $x_{i}-x_{0}=-\epsilon$. As $T_{0}$ is open, there is $\delta>0$ 
such that $\left[x_{0}-\delta, x_{0}\right] \subset T_{0}$ and for every $x \in\left[x_{0}-\delta, x_{0}\right],-\epsilon \leq x_{i}-x \leq 0$. Using full support of $\phi_{i}$, it follows that for every $x \in\left[x_{0}-\delta, x_{0}\right] \subset T_{0}, \tilde{\phi}_{0}(x) \phi_{i}\left(x_{i}-x\right)>0$. Again, $f_{i}\left(x_{i}\right)=\int_{T_{0}} \tilde{\phi}_{0}(x) \phi_{i}\left(x_{1}-x\right) \lambda(d x) \geq \int_{\left[x_{0}-\delta, x_{0}\right]} \tilde{\phi}_{0}(x) \phi_{i}\left(x_{1}-x\right) \lambda(d x)>0$. Similarly, in the final case, when $\xi=-\epsilon$, we may again show that $f_{i}\left(x_{i}\right)>0$. As $x_{i}$ is arbitrary, we conclude that for every $x_{i} \in T_{i}, f_{i}\left(x_{i}\right)>0$. From this, it follows that for every $x \in T=\times_{i=0}^{N} T_{i}, f(x)=f_{0}\left(x_{0}\right) f_{1}\left(x_{1}\right) \cdots f_{N}\left(x_{N}\right)>0$, and therefore, $T \subset$ support $(f)$. Thus, support $(\tilde{\phi}) \subset \operatorname{support}(f)$.

To see that $\tilde{\eta}$ is absolutely continuous with respect to $\mu$, fix $B \in \mathcal{T}$ arbitrarily and suppose $\mu(B)=\int_{B} f(x) \lambda^{N+1}(d x)=0$. Then either $\lambda^{N+1}(B)=0$ or for $\lambda^{N+1}$-a.e. $x \in B$, $f(x)=0$. In the first case, $\lambda^{N+1}(B)=0$ implies that $\tilde{\eta}(B)=\int_{B} \tilde{\phi}(x) \lambda^{N+1}(d x)=0$. In the second case, if for $\lambda^{N+1}$-a.e. $x \in B, f(x)=0$, then $\operatorname{support}(\tilde{\phi}) \subset \operatorname{support}(f)$ implies that for $\lambda^{N+1}$-a.e. $x \in B, \tilde{\phi}(x)=0$, from which it follows that $\tilde{\eta}(B)=\int_{B} \tilde{\phi}(x) \lambda^{N+1}(d x)=0$. Consequently, $\tilde{\eta}$ is absolutely continuous with respect to $\mu$.

In fact, this proof can be extended to show that in a global game $\Gamma$ with joint information structure $\left(\left(X_{i}, \mathcal{X}_{i}\right)_{i=0}^{N}, \eta\right), \eta$ is absolutely continuous with respect to the product of its marginals. For completeness, the proof is given in the appendix. ${ }^{7}$

Theorems 1 and 2 above, in conjunction with Balder's theorem, can be used to prove existence of equilibrium in a global game, as follows.

\footnotetext{
${ }^{7}$ As a side remark, notice that the counter-example above based on the uniform joint measure on the diagonal of the unit square is ruled out here, because the support of the errors is non-trivial, and therefore, the joint measure lives on a set of positive measure with respect to the product of its marginals.
} 
Theorem 3. Every global game has an equilibrium.

Proof. Consider a global game $\Gamma$ and consider the corresponding restricted incomplete information game $\left(\left(\tilde{\mathcal{U}}_{i}, \tilde{\mathcal{S}}_{i}, T_{i}, \mathcal{T}_{i}\right)_{i=0}^{N}, \tilde{\eta}\right)$. For this incomplete information game, notice that the assumptions on payoffs guarantee that (1) $U_{i}$ is $\mathcal{T}_{0} \times \mathcal{A}$-measurable on $T_{0} \times A$, (2) $U_{i}\left(x_{0}, \cdot\right)$ is continuous on $A$ for every $x_{0} \in T_{0}$, and (3) $\left|U_{i}\right| \leq \Phi_{i}$ on $T_{0} \times A$ for some $\Phi_{i} \in \mathcal{L}_{1}\left(T_{0}, \mathcal{T}_{0}, \phi_{0}\right)$. Moreover, for every $i=0, \ldots, N, A_{i}$ is a compact metric space. Furthermore, the previous result shows that the joint measure $\tilde{\eta}$ is absolutely continuous with respect to the product of its marginals. Consequently, the conditions in Balder's theorem (1988, page 273) are satisfied. Therefore, there exists a profile of strategies $\left(\tilde{s}_{0}^{*}, \tilde{s}_{1}^{*}, \ldots, \tilde{s}_{N}^{*}\right)$ that is a Bayesian-Nash equilibrium in this restricted incomplete information game. Using theorem 1 , we may extend this profile to a global game equilibrium $\left(s_{1}^{*}, \ldots, s_{N}^{*}\right)$ in $\Gamma$.

Theorem 3 shows that there is a global games equilibrium in a large class of global games without the restriction of strategic complementarity. As interest in global games moves beyond cases with strategic complements, our result provides a general framework in which the global games method is well-defined. This provides a foundation for the study of equilibrium behavior in global games with strategic heterogeneity.

A class of global games for which existence of equilibrium is not known generally is one that has both strategic complements and substitutes. These include games that have both complementarity and congestion. Intuitively, when a smaller number of (or smaller proportion of) competitors are taking one action, it may be in a given player's best interest 
to also take that action (strategic complements), but if a large number (or proportion) of competitors are taking the same action, it may be in a given player's best interest to take the opposite action, capturing a type of congestion effect.

Karp, Lee, and Mason (2007) study a version of such a game, modeled as follows. Suppose there are finitely many players, indexed $i=1, \ldots, N,(N \geq 2,)^{8}$ each considering an action in $A_{i}=\{0,1\}$ (endowed with the discrete topology and sigma-algebra). The payoff of each player depends on an underlying state of the world $x_{0} \in \mathbb{R}$ and on the proportion of other players who play action 1 , and is given by

$$
U_{i}\left(x_{0}, a_{1}, \ldots, a_{N}\right)=\left(x_{0}+f\left(\frac{1}{N-1} \sum_{j=1, j \neq i}^{N} a_{j}\right)\right) a_{i}
$$

where $f:[0,1] \rightarrow \mathbb{R}$ is a continuous function. ${ }^{9}$ Strategic interaction can vary depending on the response of $f$ to the proportion of others playing a given action.

Dominance regions are assumed as usual. There are real numbers $\underline{x}<\bar{x}$ such that for $x_{0}<\underline{x}$ the strictly dominant action for every player is to play 0 and for $x_{0}>\bar{x}$ the strictly dominant action for every player is to play 1.

The information structure is given as follows. Let $\varepsilon_{0}$ be a random variable distributed uniformly on $X_{0}=(\underline{x}-\epsilon, \bar{x}+\epsilon)$ with density $\phi_{0}$. Notice that in this case, $X_{0}=T_{0}$. Given an underlying state of the world $x_{0}$, each player $i$ observes $x_{i}=x_{0}+\varepsilon_{i}$, where $\varepsilon_{i}$ are identically distributed uniformly on $[-\epsilon, \epsilon]$, with density $\phi_{i}$, and $\left(\varepsilon_{i}\right)_{i=0}^{N}$ are jointly independent. Finally, for each player $i=1, \ldots, N$, as $A_{i}$ are finite and $f$ is continuous, it

\footnotetext{
${ }^{8}$ Karp, Lee, and Mason (2007) assume a continuum of symmetric players.

${ }^{9}$ Karp, Lee, and Mason (2007) assume that $f$ is an analytic function, hence smooth.
} 
follows that $U_{i}$ is continuous and bounded on $X_{0} \times A$ and is integrable.

These components define a global game. By theorem 3, this finite-player version of the global game in Karp, Lee, and Mason (2007) has a global game equilibrium.

In fact, the same result holds for more general games with both complementarity and congestion. The action spaces can be compact metric spaces, the interactions are not required to be aggregative, and both the prior distribution and the error distributions can be more general.

\section{Conclusion}

We formulate a model of a global game with general payoff structures and prove existence of equilibrium in this model. This provides a foundation for the study of equilibrium behavior in more general global games, especially as research in global games moves beyond cases with strategic complements.

Our proof of existence relies on relating a global game to its corresponding incomplete information game, and further focusing on the corresponding restricted incomplete information game. As an important step in the proof, we show that in every global game, even though the information of each player is correlated, the joint information measure (either in the restricted incomplete information game or in the global game) is absolutely continuous with respect to the product of its marginals. 
As an application, our result can be used to show that an equilibrium exists in a class of global games with both complementarity and congestion. In particular, there is a global game equilibrium in the finite-player version of the complements-congestion model in Karp, Lee, and Mason (2007), thus addressing a gap in the proof of equilibrium existence documented in Hoffmann and Sabarwal (2015).

We acknowledge that the problems of limit uniqueness and noise independence remain open in the general case. Existence of equilibrium provides a first step to other researchers in this regard. 


\section{References}

Angeletos, G. M., C. Hellwig, and A. Pavan (2006): "Signaling in a global game: Coordination and policy traps," Journal of Political Economy, 114(3), 452-484.

BALDER, E. J. (1988): "Generalized Equilibrium Existence Results For Games With Incomplete Information," Mathematics of Operations Research, 13, 265-276.

Basteck, C., T. R. Daniëls, and F. Heinemann (2013): "Characterising equilibrium selection in global games with strategic complementarities," Journal of Economic Theory, 148(6), 2620-2637.

Carlsson, H., and E. Van Damme (1993): "Global games and equilibrium selection," Econometrica, 61(5), 989-1018.

Frankel, D. M., S. Morris, and A. Pauzner (2003): "Equilibrium selection in global games with strategic complementarities," Journal of Economic Theory, 108, 1-44.

Heinemann, F., R. Nagel, and P. Ockenfels (2004): "The theory of global games on test: Experimental analysis of coordination games with public and private information," Econometrica, 72(5), 1583-1599.

Hoffmann, E. J., And T. SABARwal (2015): "A global game with strategic substitutes and complements: Comment," Games and Economic Behavior, 94, 188-190.

Karp, L., I. LEE, AND R. MASON (2007): "A global game with strategic substitutes and complements," Games and Economic Behavior, 60, 155-175.

Milgrom, P. R., and R. J. Weber (1985): "Distributional Strategies For Games With Incomplete Information," Mathematics of Operations Research, 10(4), 619-632.

Monaco, A. J., and T. Sabarwal (2016): "Games with strategic complements and substitutes," Economic Theory, 62(1), 65-91.

Morris, S., AND H. SHIN (2003): "Global games: Theory and applications," in Advances in Economics and Econometrics. Proceedings of the Eighth World Congress of the Econometric Society, pp. 56-114. Cambridge University Press.

OuRY, M. (2013): "Noise-independent selection in multidimensional global games," Journal of Economic Theory, 148, 2638-2665. 
Roy, S., And T. SABARwal (2012): "Characterizing stability properties in games with strategic substitutes," Games and Economic Behavior, 75(1), 337-353.

Steiner, J., and C. Stewart (2008): "Contagion through learning," Theoretical Economics, 3(4), 431-458. 


\section{Appendix}

Theorem 4. Let $\Gamma$ be a global game and $\left(\left(X_{i}, \mathcal{X}_{i}\right)_{i=0}^{N}, \eta\right)$ be the corresponding joint information structure. The joint measure $\eta$ is absolutely continous with respect to the product of its marginals.

Proof. Recall that $\eta(B)=\int_{B} \phi(x) \lambda^{N+1}(d x)$, where $\phi\left(x_{0}, x_{1}, \ldots, x_{N}\right)=\phi_{0}\left(x_{0}\right) \phi_{1}\left(x_{1}-\right.$ $\left.x_{0}\right) \cdots \phi_{N}\left(x_{N}-x_{0}\right)$ on $X=\times_{i=0}^{N} X_{i}, \lambda^{N+1}$ is Lebesgue measure on $\mathbb{R}^{N+1}$ and $B \in \mathcal{X}$. For each $i=0,1, \ldots, N$, let the marginal density on $X_{i}$ derived from $\phi$ be given by $f_{i}\left(x_{i}\right)=\int \phi\left(x_{i}, x_{-i}\right) \lambda^{N}\left(d x_{-i}\right)$, where $\lambda^{N}$ is Lebesgue measure on $\mathbb{R}^{N}$, and the corresponding marginal measure is given by $\mu_{i}(B)=\int_{B} f_{i}\left(x_{i}\right) \lambda(d x)$, where $\lambda$ is Lebesgue measure on $\mathbb{R}$ and $B \in \mathcal{X}_{i}$. Let the product of the marginal measures be denoted $\mu=\mu_{0} \times \mu_{1} \times \cdots \times \mu_{N}$ and the product of the marginal densities be denoted $f(x)=f_{0}\left(x_{0}\right) f_{1}\left(x_{1}\right) \cdots f_{N}\left(x_{N}\right)$. Thus, $\mu(B)=\int_{B} f(x) \lambda^{N+1}(d x)$.

To see that $\eta$ is absolutely continuous with respect to $\mu$, we first show that $\operatorname{support}(\phi) \subset$ support $(f)$, as follows. It is immediate from the definition of an information structure that support $(\phi) \subset X$. The inclusion $X \subset \operatorname{support}(f)$ can be seen as follows.

By definition, for $i=0$, and for every $x_{0} \in X_{0}, f_{0}\left(x_{0}\right)=\phi_{0}\left(x_{0}\right)>0$. Moreover, it can be seen that for each player $i \in\{1, \ldots, N\}$, and for every $x_{i} \in X_{i}, f_{i}\left(x_{i}\right)>$ 0 , as follows. Fix $x_{i} \in X_{i}$ arbitrarily, and notice that Fubini's theorem implies that $f_{i}\left(x_{i}\right)=\int \phi\left(x_{i}, x_{-i}\right) \lambda^{N}\left(d x_{-i}\right)=\int_{X_{0}} \phi_{0}(x) \phi_{i}\left(x_{1}-x\right) \lambda(d x)$. As $x_{i} \in X_{i}$, let $x_{0} \in X_{0}$ and $\xi \in[-\epsilon, \epsilon]$ be such that $x_{i}=x_{0}+\xi$. As case 1 , suppose $\xi \in(-\epsilon, \epsilon)$. In this case, $\left|x_{i}-x_{0}\right|<\epsilon$. As $X_{0}$ is open and absolute value is a continuous function, there is $\delta>0$ such that $\left[x_{0}-\delta, x_{0}+\delta\right] \subset X_{0}$ and for every $x \in\left[x_{0}-\delta, x_{0}+\delta\right],\left|x_{i}-x\right|<\epsilon$. In other words, for every $x \in\left[x_{0}-\delta, x_{0}+\delta\right] \subset X_{0}, x_{i}-x \in[-\epsilon, \epsilon]$. Using full support of $\phi_{i}$, it follows that for every $x \in\left[x_{0}-\delta, x_{0}+\delta\right] \subset X_{0}, \phi_{0}(x) \phi_{i}\left(x_{i}-x\right)>0$. Consequently, $f_{i}\left(x_{i}\right)=\int_{X_{0}} \phi_{0}(x) \phi_{i}\left(x_{1}-x\right) \lambda(d x) \geq \int_{\left[x_{0}-\delta, x_{0}+\delta\right]} \phi_{0}(x) \phi_{i}\left(x_{1}-x\right) \lambda(d x)>0 . \quad$ As case 2 , suppose $\xi=-\epsilon$. In this case, $x_{i}-x_{0}=-\epsilon$. As $X_{0}$ is open, there is $\delta>0$ such that 
$\left[x_{0}-\delta, x_{0}\right] \subset X_{0}$ and for every $x \in\left[x_{0}-\delta, x_{0}\right],-\epsilon \leq x_{i}-x \leq 0$. Using full support of $\phi_{i}$, it follows that for every $x \in\left[x_{0}-\delta, x_{0}\right] \subset X_{0}, \phi_{0}(x) \phi_{i}\left(x_{i}-x\right)>0$. Again, $f_{i}\left(x_{i}\right)=\int_{X_{0}} \phi_{0}(x) \phi_{i}\left(x_{1}-x\right) \lambda(d x) \geq \int_{\left[x_{0}-\delta, x_{0}\right]} \phi_{0}(x) \phi_{i}\left(x_{1}-x\right) \lambda(d x)>0$. Similarly, in the final case, when $\xi=-\epsilon$, we may again show that $f_{i}\left(x_{i}\right)>0$. As $x_{i}$ is arbitrary, we conclude that for every $x_{i} \in X_{i}, f_{i}\left(x_{i}\right)>0$. From this, it follows that for every $x \in X=\times_{i=0}^{N} X_{i}, f(x)=f_{0}\left(x_{0}\right) f_{1}\left(x_{1}\right) \cdots f_{N}\left(x_{N}\right)>0$, and therefore, $X \subset \operatorname{support}(f)$. Thus, support $(\phi) \subset \operatorname{support}(f)$.

To see that $\eta$ is absolutely continuous with respect to $\mu$, fix $B \in \mathcal{X}$ arbitrarily and suppose $\mu(B)=\int_{B} f(x) \lambda^{N+1}(d x)=0$. Then either $\lambda^{N+1}(B)=0$ or for $\lambda^{N+1}$-a.e. $x \in B$, $f(x)=0$. In the first case, $\lambda^{N+1}(B)=0$ implies that $\eta(B)=\int_{B} \phi(x) \lambda^{N+1}(d x)=0$. In the second case, if for $\lambda^{N+1}$-a.e. $x \in B, f(x)=0$, then support $(\phi) \subset \operatorname{support}(f)$ implies that for $\lambda^{N+1}$-a.e. $x \in B, \phi(x)=0$, from which it follows that $\eta(B)=\int_{B} \phi(x) \lambda^{N+1}(d x)=0$. Consequently, $\eta$ is absolutely continuous with respect to $\mu$. 